\title{
Prevención del Cáncer Colorrectal
}

El cáncer colorrectal (CCR) es un problema de salud importante en los países occidentales ${ }^{1}$. En España representa aproximadamente el $15 \%$ de la incidencia de todos los tumores, registrándose más de 25.000 casos nuevos al año. Considerando conjuntamente ambos sexos, es el cáncer que presenta una mayor incidencia. El CCR es más frecuente en la población de edad avanzada y su incidencia aumenta de forma importante a partir de los 50 años. En cuanto a mortalidad, es la segunda causa de muerte por cáncer, provocando más de 13.000 muertes por año. En los últimos años la mortalidad por el CCR muestra una tendencia a la disminución en ambos sexos. La supervivencia media del CCR en nuestro país es del $48 \%$ a los 5 años del diagnóstico.

En la actualidad resulta poco discutible la necesidad de un abordaje preventivo del CCR ${ }^{1}$. La prevención del CCR constituye un campo de interés común a profesionales tanto de atención primaria como de la atención especializada (gastroenterología, cirugía, oncología médica, radioterapia médica y genética, entre otras) y de salud pública. Existe un amplio consenso sobre la necesidad de concienciar a la población, a los profesionales de la salud y a las autoridades sanitarias de la importancia de la prevención del CCR.

En los últimos años se ha avanzado significativamente en el conocimiento de los mecanismos que participan en la génesis y desarrollo del CCR, lo que ha generado diferentes estrategias preventivas que pueden contribuir a disminuir la elevada incidencia y mortalidad de esta enfermedad ${ }^{1}$. Desde todos los ámbitos se deberían difundir las medidas de prevención primaria (estilos de vida saludables) y de cribado.

Dentro del Programa de "Elaboración de Guías de Práctica Clínica en Enfermedades Digestivas, desde la Atención Primaria a la Especializada" se ha publicado una segunda versión de la Guía de Práctica Clínica de Prevención del Cáncer Colorrectal ${ }^{2}$ en la cual se ha actualizado la evidencia científica disponible y se han puesto al día las recomendaciones sobre prevención primaria, cribado del CCR en la población de riesgo medio y los cribados en la poliposis adenomatosa familiar, cáncer hereditario no asociado a poliposis, CCR familiar, vigilancia de los adenomas colorrectales y en la enfermedad inflamatoria intestinal.

EI CCR muestra una fuerte asociación con los estilos de vida occidentales ${ }^{2,3}$. Durante las últimas dé- cadas diferentes estudios han demostrado que la ingesta de carne roja y procesada y los azucares, granos y almidón altamente refinados incrementan el riesgo de CCR. La sustitución de estos alimentos por carne blanca (pollo), pescado y vegetales como primera fuente de proteínas, grasas insaturadas como primera fuente de grasa, y grano no refinado y legumbres y frutas como primera fuente de carbohidratos, probablemente disminuyen el riesgo de $\mathrm{CCR}^{2,3}$. Asimismo, los suplementos de calcio podrían ser modestamente beneficiosos, y quizás también la vitamina D, B6 y el folato ${ }^{2,3}$. En cuanto a los estilos de vida, evitar el tabaco y el alcohol, prevenir el sobrepeso y la obesidad y mantener un nivel razonable de ejercicio físico se asocian claramente a una disminución del riesgo ${ }^{2,3}$. Algunos medicamentos, como la AAS, los AINES y el tratamiento hormonal sustitutivo, han demostrado una disminución del riesgo, pero su uso no está justificado en la población de riesgo medio ${ }^{2,3}$.

Estas recomendaciones de prevención primaria podrían ser un complemento del cribado del CCR para reducir la incidencia de este cáncer. El CCR cumple las condiciones necesarias para que sea susceptible de cribado ya que se conoce su historia natural, se dispone de pruebas de cribado y métodos diagnósticos con eficacia demostrada que son aplicables a la población sana sin generar riesgos excesivos, y su tratamiento en fases precoces mejora el pronóstico de la enfermedad ${ }^{2}$. Además, es una intervención que tiene una buena relación coste-efectividad².

Para realizar el cribado de CCR en individuos mayores de 50 años sin factores de riesgo adicionales se han planteado diversas pruebas: test de $\mathrm{SOH}$ bioquímico o inmunológico anual o bienal, sigmoidoscopia cada 5 años, colonoscopia cada 10 años $^{2}$. En la actualidad, existe un amplio debate por parte de las sociedades científicas de prestigio y guías de práctica clínica sobre cuál es la mejor prueba y con qué periodicidad se debería realizar. Las directrices del Consejo de la Unión Europea y el Plan Integral del Cáncer recomiendan la aplicación de un cribado poblacional de CCR con sangre oculta en heces (SOH) en hombres y mujeres de 50 a 74 años.

Durante el año 2008 la UE ha publicado un primer informe sobre el seguimiento de las recomendaciones de cribado del CCR, en el que consta que 19 de los 27 estados miembros han aceptado incorporar un programa de salud pública de cribado del CCR ${ }^{4}$. Uno de los aspectos más relevantes es la gran he- 
terogeneidad entre los distintos programas estatales. De los 19 estados miembros que se encuentran en la fase de implantación de un programa de cribado del CCR, 12 (Bulgaria, República Checa, Finlandia, Francia, Hungría, Letonia, Portugal, Rumania, Eslovenia, España, Suecia y Reino Unido) han escogido la prueba de $\mathrm{SOH}$ recomendada por la UE, 6 (Austria, Chipre, Alemania, Grecia, Italia y República Eslovaca) utilizan tanto la $\mathrm{SOH}$ como las pruebas endoscópicas, mientras que Polonia es el único estado miembro que tiene implantado un programa de cribado nacional que utiliza la colonoscopia. Actualmente 5 estados manifiestan tener en funcionamiento un programa nacional: Francia, Finlandia, Reino Unido ( $\mathrm{SOH}$ ), Italia ( $\mathrm{SOH}$ y sigmoidoscopia) y Polonia (colonoscopia).

En España la Estrategia en Cáncer del Sistema Nacional de Salud, de 2009, señala como objetivos en relación al $\mathrm{CCR}^{5}$ los siguientes: la detección precoz de cáncer de colon y recto, mediante programas de cribado para población de riesgo medio-bajo organizados con carácter poblacional a una población diana de 50 a 69 años (en una segunda etapa se podría plantear la ampliación hasta los 69 años) utilizando el test de $\mathrm{SOH}$ cada dos años. Se pretende una cobertura del $50 \%$ en este grupo de edad para el año 2015. Se hace hincapié en la necesidad de establecer sistemas de vigilancia y evaluación de calidad, a través de la Red de Programas de Cribado de Cáncer de España, impulsando el desarrollo y puesta en marcha de sistemas de información que permitan la evaluación conjunta y por CC.AA., tanto del proceso en sí como del impacto de los programas de acuerdo a los estándares establecidos en las guías de control de calidad europeas. Para las personas con riesgo elevado (pólipos adenomatosos considerados de alto riesgo o enfermedad inflamatoria del intestino) se organizarán programas de seguimiento específico. También se promoverá la evaluación del riesgo familiar de cáncer, incluyendo la indicación de realización de estudio y consejo genético de aquellas personas que cumplan criterios de riesgo de cáncer hereditario para aquellos síndromes para los que estén disponibles pruebas diagnósticas y tengan aplicabilidad clínica (síndrome de cáncer de colon no polipósico y poliposis adenomatosa familiar).

Tres CCAA (Cataluña, Murcia y La Comunidad Valenciana) han sido pioneras en disponer de un programa de cribado del CCR, aunque la mayoría de las CCAA están haciendo propuestas en este sentido. La participación en estos programas y las tasas de seguimiento son bajas e inferiores a las de otros programas de prevención del cáncer. Los motivos de esta baja participación en parte son debidos al desconocimiento de la importancia de los programas de prevención y cribado de CCR, tanto por parte de la población como de algunos profesionales sanitarios, y a la percepción de que las explo- raciones que se realizan son dolorosas y molestas.

Con el fin de divulgar la importancia del CCR, y promocionar las medidas encaminadas a su prevención, diversas sociedades científicas, entre ellas la Sociedad Española de Medicina Familiar y Comunitaria (semFYC), han unido sus esfuerzos para constituir la Alianza para la Prevención del Cáncer de Colon en España ${ }^{6}$. Las sociedades científicas y las instituciones que hemos constituido la Alianza para la Prevención del Cáncer de Colon en España consideramos que muchos de los casos de CCR pueden prevenirse y que ha de existir un elevado grado de consenso sobre la necesidad de instaurar programas de cribado del CCR en la población de riesgo medio. Es importante sensibilizar a la población de los beneficios de la prevención y el diagnóstico precoz y facilitar su acceso a los recursos sanitarios. Iniciativas como la organización del día de la prevención del cáncer ayudaran a concienciarnos a todos sobre la importancia de esta enfermedad que sin duda puede prevenirse. Los profesionales de atención primaria y especializada han de ser un claro referente para la población.

Mercè Marzo, Begoña Bellas, Elena Melús,
Marian Nuin, Carmen Vela, Mercè Vilarrubí
Grupo de Prevención del Cáncer del PAPPS
de semFYC

\section{BIBLIOGRAFÍA}

1. Marzo Castillejo M, Bellas Beceiro B, Melus Palazón E, Nuin Villanueva M, Vela Vallespín C, Vilarrubi Estrella M. Prevención del càncer. Programa de actividades preventivas y de promoción de la salud (PAPPS). http://www.papps.org/. Consultado 12 de junio de 2010.

2. Grupo de trabajo de la guía de práctica clínica de prevención del cáncer colorrectal. Actualización 2009. Guía de práctica clínica. Barcelona: Asociación Española de Gastroenterología, Sociedad Española de Medicina de Familia y Comunitaria, y Centro Cochrane Iberoamericano; 2009. Programa de Elaboración de Guías de Práctica Clínica en Enfermedades Digestivas, desde la Atención Primaria a la Especializada: 4. www.guiasgastro.net/guias_full/textos/ccolon.pdf. Consultado 12 de junio de 2010.

3. Chan AT, Giovannucci EL. Primary prevention of colorectal cancer. Gastroenterology. 2010; 138:2029-2043.

4. Grau J, Serradesanferm A, Polbach S, García-Basteiro AL, Trilla A, Castells A. Colorectal cancer screening programs in the population at average risk in the European Union and Spain. Gastroenterol Hepatol. 2010; 33:111-8. Epub 2009 Jun 11.

5. Estrategia en Cáncer del Sistema Nacional de Salud, de 2009. Ministerio de Salud y Política Social. Plan de Calidad para el Sistema Nacional de Salud.

6. Alianza para la Prevención del Cáncer de Colon. http://www. alianzaprevencioncolon.es/. Consultado 12 de junio de 2010. 